\title{
EVERY MORNING YOU HAVE TO WAKE UP AND BE YOU? PSICOPATÍA Y ACTANCIA EN GONE GIRL DE GILLIAN FLYNN (2012)
}

\author{
Elena Avanzas Álvarez, Universidad de Oviedo ${ }^{3}$ \\ Email: eleavanzas@gmail.com
}

\begin{abstract}
Resumen: Gone Girl de Gillian Flynn se ha convertido en una de las más exitosas y conocidas novelas de crímenes de los últimos años. Pero, ¿por qué? Este artículo explorará el personaje de Amy Elliott Dunne como mujer psicópata que ha revertido y cuestionado las creencias sociales de género, psiquiátricas y de actancia para las mujeres. Amy será analiza utilizando textos psiquiátricos canónicos, una narración autobiográfica y los enfoques más innovadores de la psicopatía para probar que, al terminar la novela, se ha convertido en la única persona capaz de definir su identidad, su cuerpo y su vida. Palabras clave: femenina, psicopatología, psiquiatría, cuerpos, problemas de género, crimen, ficción, contemporánea, literatura.
\end{abstract}

Título en inglés: "Every morning you have to wake up and be you?" Psychopathy and Agency in Gillian Flynn's Gone Girl (2012).

Abstract: Gone Girl by Gillian Flynn has become one of the most successful and wellknown crime fiction books in recent years. But why? This article explores the character of Amy Elliott Dunne as a female psychopath who has managed to subvert and question gender, psychiatry and agency expectations for women. Amy will be analysed using canonical psychiatric texts, a biographical account and the most innovative approaches to psychopathy to prove that by the end of the novel she emerges as the only dominant force in her life, her body and her identity.

Keywords: female, psychopathology, psychiatry, human bodies, gender issues, crime, fiction, contemporary, literature.

\section{INTRODUCCIÓN}

Gillian Flynn publicó su tercera novela, Gone Girl, en junio de 2012 con la que la autora norteamericana ha logrado cambiar la literatura de crímenes para siempre. Desde entonces, la frase "The next Gone Girl" se ha convertido en una herramienta de marketing en la industria literaria. Como Ianzito bien señala en su artículo "The New 'Gone Girl'? You Decide":

Date of reception:29 October 2014

Date of acceptance: 10 November 2014 
Everybody's looking for the next Gone Girl-publishers, who want to mimic the success of Gillian Flynn's summer 2012 thriller that sold more than six million copies, and readers, who gobbled up every word (weak ending and all). No surprise, then, that publishers and industry-watchers have hyped any number of books as "the next Gone Girl" (2013).

El éxito fue tal, que sólo unos meses tras su publicación, los derechos del libro se subastaron para crear una adaptación fílmica. La película, con el mismo título que el libro, dirigida por David Fincher, con Ben Affleck como Nick y Rosamund Pike como Amy, llegó a las pantallas en octubre de 2014 y se ha convertido en un éxito inmediato ${ }^{4}$. La pregunta que hay que hacerse, entonces, es: ¿Qué hace especial a Gone Girl?

Gone Girl se divide en tres grandes apartados: "Boy Loses Girl", donde Amy ha desparecido y Nick es presentado como sospechoso del crimen que acabó con la vida de Amy; "Boy Meets Girl", donde Amy finalmente confiesa que está viva y su desaparición es un intento de acusar falsamente a Nick de su asesinato y, finalmente, "Boy Gets Girl Back (Or Vice Versa)", donde Amy regresa a casa y retoma su relación con Nick. Este artículo se centrará principalmente en la segunda parte, ya que es en ella donde Amy se configura como una psicópata y donde cuestiona la moralidad del público deconstruyendo y subvirtiendo las normas de comportamiento normalizado. Este análisis la considerará un sujeto agente empoderado por sus acciones quien, además, demuestra que la novela de crímenes se ha convertido en un tex to fluido y en una herramienta de negociación de valores morales.

La metodología a utilizar se basará en textos canónicos de psiquiatría moderna, una narración autobiográfica, un análisis Foucaldiano y teorías corporales para definir a Amy como una psicópata y, a la vez, demostrar que no es una enferma mental. Las teorías estarán ejemplificadas con citas de la novela y complementadas con otros textos que ofrecen una perspectiva innovadora o rompedora de la mujer psicópata en la sociedad y la literatura de crímenes producida en el siglo XXI.

Finalmente, el objetivo de este artículo es analizar el arco identitario que Amy Elliott Dunne crea en Gone Girl y cómo, tras una serie de experiencias, logra construirse como sujeto agente desafiando las construcciones de género de la sociedad moderna. En este proceso utilizará herramientas como la ficción, la re-escritura de su pasado, la plasticidad de su cuerpo y jugará con lo esperado de cada género en contextos criminales.

\section{PSICOPATÍA FEMENINA}

En Masquerade, Crime and Fiction: Criminal Deceptions (2006), Linden Peach explora, en el capítulo "Where Does That Criminality Come From? Writing Women and Crime", la relación que hay entre las mujeres y el crimen. El primer papel que las mujeres jugaron en la literatura de crímenes a través de sus cuerpos, fue el rol de víctimas, mientras que el detective, siempre en masculino, simbolizaba el orden y la moralidad. Sally Munt en Murder by the Book? habla de cómo el detective "represents the paraonaia of dominant hegemonic Christian/patriarchal order" (1994: 3), mientras que lo femenino adopta el rol de víctima pasiva. Pero, en la literatura del siglo XXI, el papel de las mujeres en este género

\footnotetext{
4 Gone Girl (2014) recaudó 37.513.109 de dólares sólo en el fin de semana de su estreno en EE.UU (3.014 pantallas).
} 
literario ha evolucionado hasta convertirlas en detectives y en criminales, pero, sobre todo, en protagonistas. Gone Girl es una novela de crímenes, porque la narración comienza con la desaparición de Amy y las sospechas, tanto de la detective asignada al caso como del público lector, de que Nick la ha matado. Los roles tradicionales de investigadora, víctima, y criminal serán re-asignados durante la narración para así cuestionar si las acciones de Amy, legalmente criminales, aunque justificadas en el texto, la convierten o no, en una criminal. Peach explora cómo el status de las mujeres en relación con la criminalidad emerge en ciertos contextos: "[i]nterest in female criminality seems to be the most pronounced at times of significant change in the cultural representation and the status of women" (Peach 2006: 81), contextualizando Gone Girl y explicando el éxito que ha tenido en el momento de acusada crisis económica, que ha obligado a redefinir los roles de género, en el que texto salió a la luz.

La psicopatía es una conducta que se compone de un conjunto de rasgos, como bien demuestra The Psychopathy Checklist, creada por el doctor Robert Hare. El doctor John F. Edens, en la evaluación psicológica que precede a la autobiografía de E.M Thomas, Confessions of a Sociopath (2013), describe algunos de estos rasgos tales como tendencias antisociales, egocentrismo, alta auto-estima, búsqueda de atención excesiva, agresión verbal y dominancia interpersonal, además de falta de experiencia con emociones altamente negativas como traumas o fobias (Thomas 2013: Psychological Evaluation Excerpt). Esta descripción encaja perfectamente con el personaje de Amy y sus acciones en Gone Girl. Además, la característica más importante de Amy es su alto nivel de adaptación social que le permite camuflarse de forma camaleónica para conseguir el beneficio propio. Especialmente por este último rasgo, hay que considerar a Amy una psicópata, o una persona con rasgos psicopáticos muy desarrollados, ya que es necesario subrayar que la psicopatía no se trata de un diagnóstico absoluto, sino gradual.

El mismo Hare, señala que los rasgos psicopáticos están distribuidos entre aquello que se entiende como "población normal" (Hare, 1998). Al establecer esta dualidad, se diferencia entre aquellas personas que muestran rasgos psicopáticos, denominados "psicópatas" y entendidos como una desviación de la normalidad y el resto de la población. El sociólogo austriaco Erikson explica que lo socialmente desviado, marca los límites externos por los que la sociedad debe definirse y, por tanto, crea un contraste que le da profundidad a la norma y a lo normativo $(1966,27)$. Es decir, al hablar de psicopatía, se crea un perfil psicológico con unos rasgos que son la antítesis del comportamiento normalizado a la vez que se convierte la conducta psicopática en una conducta patológica ${ }^{5}$. Se trata de un uso intra-social de la estrategia que Edward Said denominaba la creación del "Otro": "The construction of identity [...] involves establishing opposites and 'others' whose actuality is always subject to the continuous interpretation and re-interpretation of their differences from 'us'. Each age and society re-creates its 'Others"” (Said 2003, 332). La filósofa feminista Simone de Beauvoir ya había utilizado esta dicotomía anteriormente y con perspectiva de género al hablar de la mujer como Otra, en su canónica obra El Segundo Sexo: "La mujer se determina y se diferencia con relación al hombre, y no este con relación a ella; la mujer es lo inesencial frente a lo esencial. Él es el Sujeto, él es lo Absoluto; ella es lo Otro" (1979: 12).

5 El DSM (Diagnostic and Statistical Manual of Mental Disorders), editado por la Asociación Norteamericana de Psiquiatría, en su versión V, cataloga la psicopatía como "desorden de la personalidad antisocial” (2013: 659). 
Entonces, si los psicópatas son los Otros y las mujeres, al mismo tiempo, también sufren este proceso de otredad, ¿Qué ocurre cuando una mujer es psicópata?

Es difícil encontrar literatura sobre mujeres psicópatas, como bien ejemplifican Federman, Holmes y Jacob en su artículo "Deconstructing the Psycopath" (2009): "the psychopath is almost always male" (2009: 48). De hecho, históricamente, su figura se ha comparado a la del "malo" en los Westerns (2009: 36) y esta ha evolucionado de monstruo a sujeto peligroso para, finalmente, ser catalogado en el discurso médico-legal como psicópata (2009: 57). No queda, entonces, en el discurso que ha construido esta nueva categoría social, sitio para localizar una configuración femenina de la psicopatía. En relación al Western y a la frontera, es necesario mencionar a Cathy Ames, de la novela East of Eden (1952) de John Steinbeck. Desde su primera aparición, Cathy muestra rasgos psicopáticos, pero Steinbeck la caracteriza como el mal mismo y, finalmente, recibe su merecido. Por tanto, aunque la inclusión de Cathy en la narración es una innovación en tanto que es una de los primeros personajes femeninos psicópatas, Steinbeck no explora su personalidad más allá de su construcción como un monstruo desde que la presenta: "I believe there are monsters born in the world to human parents" (1952 (2012): 89).

En el anteriormente mencionado artículo, Federman et all afirman que "[t]he key to understanding the psychopath is his behavioral deviation from the norm" (2009: 40), idea que pone de relieve la existencia de una norma de comportamiento donde también intervienen los roles de género. Es importante señalar que las características que configuran un perfil psicopático están culturalmente asociadas a rasgos masculinos que se basan en una percepción esencialista del género, tales como mayor violencia, egoísmo y falta de capacidad para cuidar de los demás. Mientras que una definición esencialista de las mujeres las colocaría en el extremo contrario del espectro psicopático, mostrando sensibilidad, atención a la familia y a la crianza y altruismo. Pero, la psicopatía también necesita de contexto geográfico, ya que esta personalidad está localizada, no sólo dentro del llamado "mundo desarrollado", sino más específicamente, dentro de los Estados Unidos, dónde el capitalismo y el mundo corporativo premian a aquellas personas que exhiben una serie de rasgos que se asemejan en extremo a los psicopáticos. Hare señala que "society is moving in the direction of permitting, reinforcing, and in some instances actually valuing some of the traits listed in the Psychopathic Checklist" (1996: 177). E.M. Thomas, en su autobiografía, refuerza este argumento al afirmar que "[i]f charm, arrogance, cunning, callousness, and hyper-rationality are considered psychopathic traits, it's probably no surprise that many sociopaths end up as successful corporate types" (2013: 12). Por tanto, teniendo en cuenta el género, tanto de las autoras como de las protagonistas de sus obras, Gone Girl y Confessions of a Psychopath demuestran una evolución con perspectiva de género del concepto de psicopatía en la Norteamérica actual.

Al tratarse de una mujer psicópata, la mascarada es una técnica de adaptación social que permite a Amy involucrarse en su entorno y, al mismo tiempo, manipular y utilizar a las personas que la rodean. Cabe señalar que la falta de empatía y conciencia social de las personas psicópatas les llevan a buscar formas de integrarse en la sociedad, haciendo que la mascarada, una simple imitación de la conducta puesta en escena, sea la más exitosa. Amy crea una personalidad ficticia, "Diary Amy, who is a work of fiction" (Flynn 2012: 220), quien sólo existe en su diario y quien se configura en oposición a su verdadera 
personalidad, Actual Amy (Flynn 2012: 220). De esta forma, se muestra la construcción de la identidad como performativa y discursiva a través del lenguaje. Por otro lado, Amy ejemplifica la teoría de la mascarada, expuesta por Joan Riviére en 1913 y aplicada a la literatura de crímenes por Linden Peach. Diary Amy es una obra de ficción que Amy modeló en el constructo social de "Cool Girl":

Being the Cool Girl means I am a hot, brilliant, funny, woman who adores football, poker, dirty jokes and burping, who plays videogames, drinks cheap beat, loves threesomes and anal sex, and jams hot dogs and hamburgers into her mouth like she's hosting the world's biggest culinary bang while somehow mantaining a size 2, because Cool Girls are above all hot. Hot and understanding (Flynn 2012: 222).

La descripción de esta conducta tan reglada y restrictiva deconstruye los modelos de comportamiento femenino esperados por la sociedad en el marco de una relación romántica. En un sistema patriarcal, el modelo de mujer arriba descrito exhibe comportamientos típicamente masculinos a la vez que los combina con una excesiva atención al cuerpo y a la estética, preocupaciones típicamente femeninas en dichos sistemas. El modelo de Cool Girl se basa, por tanto, en una performatividad que combina rasgos masculinos y femeninos, pero que, al fin y al cabo, crea un ideal ficticio patriarcal de mujer sometida en una relación heterosexual.

Amy reconoce la ficción de esta creación cuando admite que se basa en "a woman who has watched too many movies written by socially awkard men who'd like to believe that this kind of woman exists and might kiss them" (Flynn 2012: 222 cursiva en el original). Tanto Diary Amy como Cool Girl toman como referencia una construcción patriarcal de la feminidad difundida a través de diversas producciones culturales. Se trata de un ideal de comportamiento femenino que incluso ha sido internalizado por algunas mujeres, como Amy bien apunta: "women across the nation colluded in our degradation!" (Flynn 2012: 223).

Ya que las diversas mascaradas de Amy se basan en la imitación de la conducta, se trata de una construcción performativa de la identidad. Judith Butler explica la performatividad del género y cómo los actos, en general, contribuyen a crear nuestra identidad: ““'G]ender is an identity tenously constituted in time, instituted in an exterior space through a stylized repetition of acts" $(1990,140)$. Para Amy, Cool Girl era tan sólo una actuación, "a bit of a game" (Flynn 2012: 224) y esperaba que Nick, su marido, se percatase de que se trataba tan sólo de una mascarada: "I thought we had a wink-wink, don't ask, don't tell thing going" (Flynn 2012: 224).

\section{THE SHOW MUST GO ON: PERFORMATIVIDAD Y CORPOREIZACIÓN}

Sin embargo, la mascarada y la performatividad de Amy no se limitan a su identidad, sino que ella misma los traslada al espacio. La falsificación del lugar del crimen muestra el carácter performativo de estos lugares: al igual que existe una rutina utilizada por la ciencia forense para acercarse al lugar del crimen de forma que los procedimientos acerque lo más posible a quienes investigan a la verdad, Amy revierte esta secuencia de actos para crear una escena del crimen que inculpe a Nick. Tras hacerse un corte en el brazo que la hace sangrar pero no tanto como para perder la consciencia, inunda la cocina con su propia 
sangre y la limpia "as poorly as Nick would have done after he bashed my head in" (Flynn 2012: 220) y, además, hace del salón un escenario: "Stage the living room. Tip ottoman" (Flynn 2012: 220). Peach ya indicó la construcción histórica y social de la escena del crimen: "[T]he crime scene is the conduit of other histories too: the cultural history of crime; the history of criminal investigation and forensic science; and socio-economic history [...] All crime scenes are re-presented when they become the subject of a narrative, whether in the courtroom or the literary text" (2006: 60). Por lo tanto, el proceso que Amy sigue es tan sólo una deconstrucción de cómo la sociedad ha creado la ciencia forense y ella se aprovecha de este conocimiento para revertir el valor moral. Si bien las investigaciones criminales tienen como fin último la justicia, entendida legalmente, Amy aplica su propio código moral y crea una escena del crimen que, según sus valores e ideas, le parece justa: "It's rather extreme, framing your husband for your murder. I want you to know that I know that $[\ldots]$ All those things that spineless women say, confusing their weakness with morality [...] But it's so very necessary. Nick must be taught a lesson" (Flynn 2012: 234 cursiva en el original). Además, justifica sus acciones criminalizando la conducta infiel de Nick: "He killed my soul, which should be a crime. Actually, it is a crime. Acording to me, at least" (Flynn 2012: 238).

El relativismo moral de Amy se presenta en el texto de forma emocional y su percepción de las normas sociales y posterior justificación de sus propios actos recuerda a la forma en la que Thomas percibe la moralidad: "the heart of morality is something I have never understood" (2013: 8). Flynn hace que su personaje principal apele a los sentimientos de quien lee para producir una respuesta favorable y así hacer que la entrada a la narración se produzca a través de su focalización, que también incluye su cuerpo. Esta entrada se produce, como Munt indica, a través del cuerpo femenino: "instead of being titillated by the abused female body, we are inscribed within it, and allowed revenge" (Munt 1994: 194). En la primera parte de la novela, el cuerpo de Amy se construye como abusado a través de su ausencia y su silencio, pues la narración sugiere que Nick la ha asesinado. De esta forma, quien lee se identifica con Amy, quien, a su vez, utiliza argumentos sentimentales como técnica para seducir al público, a quien además apela directamente.

Como consecuencia, la focalización se produce a través de su punto de vista y se produce una respuesta favorable a un personaje que, hasta hace muy poco, no habría contado con simpatizantes. Roxane Gay, en su ensayo "Not Here to Make Friends" incluido en Bad Feminist (2014) trata la complejidad de los personajes femeninos que no gustan al público lector y la diferencia de percepción que hay dependiendo del género del personaje. Cuando un personaje masculino muestra una moralidad dudosa o sus acciones perjudican a otros, se le considera un anti-héroe. Sin embargo, cuando un personaje femenino exhibe estas mismas características, se convierte en un problema para la crítica: "When women are unlikable, it becomes a point of obsession in critical conversations by professional and amateur critics alike" (2014: 88). En muchos casos, el mal personaje femenino necesita una patología que muestre los inicios y la causa de su desviación de, lo que Gay llama, el mundo ideal que esperamos que sea la ficción: "Pathologizing the unlikable in fictional characters is an almost Pavlovian response" (2014: 91). Sin embargo, el comportamiento altamente adaptativo de Amy, como consecuencia de su psicopatía, no debe considerarse una patología entendida como enfermedad mental. Es decir, sus acciones la hacen adaptarse 
al entorno de forma camaleónica para sacar el mayor beneficio posible del contexto en el que se encuentra. Una conducta que, extremadamente simplificada, ejemplifica la idea de "the survival of the fittest" de la que hablaba Charles Darwin. También Thomas aboga por separar la conducta psicopática de la enfermedad mental: "I may have a disorder, but "I am not crazy" (2013: 3). Además, al tratarse de un personaje, es necesario desvincular su identidad como psicópata de la construcción de "enferma mental" que tanto ha restringido y silenciado a las mujeres históricamente.

En el ensayo anteriormente citado Gay dedica unas palabras a Amy "[p]erhaps the most unlikable woman in recent fictional memory" (2014: 94), la describe como un personaje repelente y antipático y se refiere a la narración como incómoda (2014: 94). Pero, sobre todo, presta atención, a las muchas faltas que tanto Amy como Nick tienen y que hacen de Gone Girl, "a character study" (2014: 94) del que Amy es la indiscutible protagonista. Gay también elogia la valentía de Flynn al crear un personaje femenino que no está sometido a la tiranía del mundo ideal que, a veces, se espera que sea la ficción. Amy es una de esas mujeres protagonistas que expone los prejuicios sociales y la construcción del género que se esconden tras la falacia del "personaje femenino que gusta", es decir, que se muestra favorable a las convenciones sociales. A su vez, destapa la verdadera complejidad y riqueza de aquellos personajes que: "[ $\mathrm{t}]$ hey are, insated, themselves. They accept the consequences of their choices, and those consequences become stories worth reading" (Gay 2014: 95).

Una de las mayores herramientas sugestivas de Gone Girl es el tono confidencial que Amy adopta a través de la narración en primera persona y su diálogo directo con el público lector. "Can you imagine, finally showing your true self to your spouse, your soul mate, and having him not like you?" (Flynn 2012: 225) pregunta directamente a quien lee en el capítulo donde desvela que no está muerta y su del cuerpo es hasta que no comete un acto psicopanterior que no fuese construida en una criminal.plan es que acusen falsamente a Nick. Este tren de pensamiento sigue con Amy justificando sus acciones mientras re-escribe la historia que ha sido narrada en la primera parte del libro. Amy desmonta a Diary Amy y deja de ser una víctima para tomar las riendas de la situación y hacer ver su punto de vista: "So, that's how the hating first began. I've thought about this a lot, and that's where it started, I think" (Flynn 2012: 225), explica. A través de "I think", Flynn está utilizando el texto para jugar con la percepción que el público tiene de Amy, quien ya se ha desvelado como una artífice de la mascarada. Peach establece, "The detective novel is basically a game between the author and the reader" (2006: 57) y en Gone Girl, Amy engaña al público lector durante la primera parte de la novela para, ya en la segunda, hacernos cómplice de su plan.

Al tratarse de una mujer psicópata, es necesario analizar la feminidad de Amy a lo largo de la narración. Stephen Heath en "Joan Riviere and the Masquerade" (1986) afirma que la auténtica feminidad es esencialmente una mascarada y que no existe una feminidad original $y$, por tanto, anterior. Butler también sostiene este argumento afirmando que no existe un original para el sujeto, sino un conjunto de actos que, finalmente, constituyen la identidad:

The subject is not determined by the rules through which it is generated because signification is not a founding act, but rather a regulated process of repetition that both conceals itself and enforces its rules precisely through the production of substantializing effects (1990: 145). 
Flynn presta especial atención a cómo los padres de Amy jugaron un papel importante en la creación de su identidad y explora cómo, para la protagonista, no hubo una feminidad anterior que no fuese construida. Amy fue un milagro médico tras cinco abortos y dos partos de fetos muertos, de tal forma que creció compitiendo contra "seven dead dancing princesses" (Flynn 2012: 222), una infancia que ella describe como: "[A]n exhausting way to live" (Flynn 2012: 222). De esta forma, la experiencia vivida de la infancia de Amy, que probablemente la llevó a desarrollar su carácter hiper-competitivo, crea el entorno perfecto para que florezca una personalidad psicopática. Se ven trazas de esta personalidad cuando ella misma reconoce su incapacidad para simpatizar con otras niñas durante su infancia: "I would be at a birthday party and watch the other kids giggling and making faces, and I would try to do that too, but I wouldn't understand why" (Flynn 2012: 224 énfasis en el original).

Ya que Amy utiliza la ficción en su vida para crear adaptaciones al entorno que la beneficien, es necesario analizar cómo se presenta esta ficción a través del texto en Gone Girl. Flynn lo hace a través de dos construcciones: Diary Amy y Amazing Amy. Como Diary Amy ya ha sido discutida anteriormente, es necesario ahora prestar atención a Amazing Amy, el personaje protagonista de una serie de libros infantiles creados por los padres de Amy e inspirados en la propia niña. La ficcional Amazing Amy triunfaba en todo aquello en lo que la verdadera Amy competía y fracasaba: "My parents have always worried that I'd take Amy too personally - they always tell me not to read too much into her. And yet I can't fail to notice that whenever I screw something up, Amy does it right; When I finally quite violin at age twelve, Amy was revealed as a prodigy in the next book" (Flynn 2012: 26). Como personaje de ficción, Amazing Amy estaba totalmente a merced de los Elliott, de forma que no tenía un cuerpo a través del cual realizar acciones que la definiesen y, además, le otorgasen actancia ${ }^{6}$. La oposición entre la aséptica y maleable Amazing Amy y la corporeización de Amy Elliott presenta uno de los mayores problemas que el feminismo se ha encontrado en sociedades patriarcales: la necesidad de controlar y someter el cuerpo femenino.

Este conflicto entre realidad y ficción se refleja en el cuerpo de Amy desde el momento en que nace, ya que los anteriores fracasos reproductivos de los Elliott los llevaron a considerar a Amy una sorpresa ante la que no supieron cómo reaccionar. No tenían pensado un nombre para ella, porque los anteriores fetos había llevado del nombre simbólico de "Hope" y, para su única hija viva, escogieron Amy porque se trataba de un nombre común, una decisión que la marcará de por vida. La disonancia entre el significante, el nombre común que "a thousand other baby girls were given that year" (Flynn 2012: 221) y el significado, "I grew up feeling special, proud. I was the girl who battled oblivion and won. The chances were about 1 percent" (Flynn 2012: 221), muestra la lucha de Amy contra lo esperado de ella y lo construido. Además, Amazing Amy, como personaje, no tiene cuerpo, mientras que la Amy real tiene un cuerpo que a la vez simboliza todos aquellos cuerpos anteriores que no sobrevivieron. Cuando Amy nace, su madre pregunta constantemente: "Is she still alive?" (Flynn 2012: 221) y, cada otoño en el aniversario de los abortos y los embarazos fallidos, Amy, a través de su cuerpo, es rechazada por su madre: "she would get pensive, she would remove herself, and I would have none of it, needful thing that I was" (Flynn 2012: 221).

\footnotetext{
6 "Actancia” es la traducción más correcta de "agency", pues en el original en inglés los actos son definitorios.
} 
En el último volumen de la serie, los Elliott le han dado a la Amazing Amy algo que no han podido a la real, un marido, y que, entonces, Amy se encarga de conseguir por ella misma. M.E. Thomas en Confessions of a Sociopath (2013) habla de cómo "[m]ost sociopaths want to hide their identity [...] People don't like sociopaths" (Thomas 2013: 302), motivo por el que Amy pudo haber escondido su verdadera identidad a lo largo de su vida a través de distintas mascaradas. La estrategia de Amy al entrar en una relación con Nick encaja con el tipo de comportamiento psicopático, en tanto que sigue la misma técnica que ha aprendido desde niña: crear una mascarada, Cool Girl, que satisfaga el contexto social en el que se encuentra, en este caso, una relación heterosexual. Amy reconoce saber lo que Nick quería de ella en cuanto lo vio "When I met Nick, I knew immediately that [a Cool Girl] was what he wanted" (Flynn 2012: 223), de la misma forma que Thomas hace referencia a la capacidad de las personas psicópatas para convertirse en "a fascimile of whatever or whoever that person wants - a good employee or boss or lover" (2012: 7).

La capacidad de adaptación derivada de la psicopatía plantea cuestiones sobre la responsabilidad y la libertad de actuación, e incluso la actancia, de Amy. De acuerdo con Federman, et al [t]he idea of willful murder (or willful manipulator) without mental illness creates a space for the legal and social construction of responsability" (2009: 43), un espacio que, sin embargo, todavía no ha sido acotado y definido. Sin embargo, en toda la narrativa de Gone Girl, Amy se muestra responsable de sus actos, planeando y ejecutando su plan para inculpar falsamente a Nick como consecuencia de la aventura que éste ha tenido con una alumna. Reafirma así la teoría de Michaud y Aynesworth de que "psychopaths, often mantain responsability for their behaviour: they are their behaviour" (2009: 49). Es necesario mencionar que aunque Amy, y las personas psicopáticas en general, se hagan responsables de sus actos y se vean definidas por ellos, siempre exponen una motivación externa que les ha obligado o les ha llevado a actuar. Por tanto, si Amy es una psicópata y sus actos la definen, y esos actos son una decisión suya, planeada y orquestada, entonces cabe concluir que, como mujer psicópata, Amy es un sujeto agente y establece su actancia a través de sus actos, performados a través de su cuerpo.

Federman et al (2009) localizan las primeras psicopatías en el siglo XIX, cuando los psiquiátricos proliferaron y lograron extraer y catalogar a aquellas personas que se desviaban de la norma, a la vez que establecían claramente este comportamiento desviado como la definición en negativo de la normalidad. Pero, la psicopatía no tiene reflejo en el cuerpo, de forma que un análisis físico no demuestra diferencias con aquellas personas "normales" y la psiquiatría fue incapaz de "locate deivant behaviour within the body" (2009: 47). Esto hace que las personas psicópatas sean invisibles hasta que realizan un acto que las defina como tal y que conlleva el "mal" social: el psicópata no lo es hasta que no comete un acto psicopático. Pero, al ser Amy una mujer psicópata, está subvirtiendo no sólo la historia de la patología, sino también la definición del comportamiento como patológico, que, además, se ha mantenido estático y no ha sido adaptado los contextos sociales e históricos. Su cuerpo es, además, el reflejo, no sólo de la evolución de sus identidades, sino también de su psicopatía. Tras escapar del lugar del crimen que ella misma creó, Amy se cambia el color de pelo y escapa también del cuerpo resultado de su mascarada de Cool Girl: "I have dark skin, my mouse-colored helmet cut, the Smart-girl glasses. I gained twelve pounds in the months before my disappearance -carefully hidden in roomy sundresses" (Flynn 
2012: 250). Tras ser reconocida y acudir a su ex-novio, Desi, en busca de ayuda, Amy ve su cuerpo sometido a las normas patriarcales de belleza una vez más:

He enters and he makes my mouth water. The smell. He brings me something nice to eat, but not as nice as what he's had: He's thining me up, he always preferred his women waify $[\ldots]$ I am almost my normal weight again, and my hair is growing out [...] and I have colored it back to my blond, thanks to hair dye he also brought me (Flynn 2012: 361).

Pero, la prisión en la que Desi encierra a Amy es corporal, al obligarla a recuperar su físico anterior, y también física, ya que no le permite salir de la mansión donde se refugian. Sin embargo, tras ver una emotiva y muy manipulada entrevista a Nick en televisión, Amy decide volver con su marido y, para ello, necesita escapar de Desi. Lo seda y crea otra escena del crimen donde ella juega el papel de víctima y convierte su cuerpo en un espacio criminalizado: "I still have Desi's semen inside me from the last time he raped me, so the medical examination goes fine. My rope-weathed writs, my damaged vagina, my bruises - the body I present them is textbook" (Flynn 2012: 374). Además, abandonó la escena del crimen "still slick with his blood, into his vintage Jaguar" (Flynn 2012: 374), utilizando su cuerpo como prueba y como símbolo del crimen al que supuestamente ha sido sometida. El cuerpo de Amy se ha convertido no sólo en un arma, sino en un ente plástico que ella moldea a merced de sus deseos y sus motivaciones para infiltrarse socialmente jugando el papel que más la beneficie en ese momento.

\section{CONCLUSIONES}

Tras este análisis, cabe concluir que Amy Elliott Dunne ha creado una nueva categoría de personaje femenino en la literatura de crímenes del siglo XXI. Su carácter psicopático desafía las creencias y los estudios psiquiátricos al redefinir la imagen del psicópata como una mujer y no como un hombre, además de cuestionar la categorización patológica de su conducta. Esta característica la hace un personaje subversivo, ya que cuestiona lo típico de y lo esperado de su género en la sociedad patriarcal: Amy sigue las normas, pero no las internaliza.

Además, Amy se muestra responsable de sus actos durante toda la novela, reforzando su posición como sujeto agente y cuestionando que sufra de una patología. Los rasgos psicopáticos, categorizados en el canónico DSM, en su quinta edición, como un desorden de la personalidad, la situarían en los límites de la sociedad, en un asilo simbólico a las afueras de la ciudad. Esta marginalización ha sido configurada desde el siglo XIX como una negativa, al excluir a la persona de la sociedad. Sin embargo, Amy redefine este lugar como privilegiado ya que su actancia le permite la total integración social a través de mascaradas desde las que cuestiona las construcciones de género.

No hay duda de que Amy tiene actancia y lo demuestra en la novela a través de su historia personal. En su infancia simbolizó lo imposible y a la vez lo vulgar, de forma que sus padres crearon una Amy ficticia cuyos logros sobrepasaban los de su propia hija. Pero, ya en la edad adulta, Amy tomó las riendas de la ficción, se convirtió en autora y creó el personaje de Diary Amy. Su actancia se refleja, además, en su cuerpo: de niña fue recha- 
zada por sus padres al representar los anteriores fracasos reproductivos que precedieron su nacimiento, ya en la edad adulta, Amy sucumbió al ideal patriarcal de belleza y creó Cool Girl: rubia, delgada, sumisa y dócil. Tras desparecer, abandonó este comportamiento y, durante unas semanas, encontró la libertad para exponer su cuerpo: pelo más oscuro, piel bronceada y un aumento de peso crearon una especie de limbo corporal. Sin embargo, Desi la hace obliga a retornar al ideal patriarcal de feminidad mostrando la necesidad de control y restricción del cuerpo femenino en dinámicas heterosexuales patriarcales. Pero, en lugar de sentirse sometida y restringida, Amy revierte este discurso y utiliza su cuerpo como un arma para matar a Desi. Se agrede sexualmente a si misma con una botella y se causa las heridas necesarias para que su cuerpo refleje lo que ella quiere: ser víctima de un secuestro y una agresión sexual. No hay duda de que, al final de Gone Girl, la actancia de Amy es tal, que se traduce en la fluidez y el auto-control extremos de su cuerpo.

Es, además, a través de este que el público lector accede a la lectura de Gone Girl como una novela de crímenes, ya que es el silencio del cuerpo de Amy lo que pone la narración en marcha al sugerir que ha sido asesinada por Nick. Munt afirma que el acto de buscar y encontrar en la novela de crímenes es "a performative rather than evaluative function" (Munt 1994: 201). Por consecuencia, el cuerpo de Amy es clave para la performatividad y para que, a través de él, accedamos como público lector a la experiencia epistemológica de la lectura. Además, al realizar esta entrada a través del cuerpo de Amy, accedemos también a través de su mente y de su punto de vista, de forma que aunque sus acciones sean moralmente condenables en la vida real, en Gone Girl, son la empresa de un personaje principal que, como buena psicópata, nos ha cautivado. Amy pone de relieve el sometimiento femenino del cuerpo en la Norteamérica del siglo XXI a la vez que su psicopatía la sitúa parcialmente fuera de lo social, desde donde crea un discurso que deconstruye el ideal patriarcal de feminidad y donde su cuerpo ya no la convierte en víctima pasiva, sino que la empodera y le otorga actancia.

\section{REFERENCIAS BIBLIOGRÁFICAS}

American Psychiatric Association. 2013. Diagnostic and Statistical Manual of Mental Disorders 5. Washington: American Psychiatry Publishing.

Beauvoir, Simone de. 1979. El Segundo Sexo. Madrid: Debolsillo.

ButLER, Judith. 1990. Gender Trouble: Feminism and the Subversion of Identity. Nueva York: Routledge.

ERIKSON, KAI. 1966. Wayward Puritans: A Study in the Sociology of Deviance. Nueva York: Wiley \& Sons.

Federman, C, Homes, D, JACoB, J.N. 2009. "Deconstructing the Sociopath: A Critical Discursive Analysis". Cultural Critique 72: 36-65.

Flynn, Gillian. 2012. Gone Girl. Nueva York: Crown Publishing.

GAY, RoXANE. 2014. Bad Feminist. Nueva York: Harper Collins. 
Hare, Robert. 1996. "Pschopathy: A Clinical Construct Whose Time Has Come." Criminal Justice and Behaviour 23, 1: 25-54.

Hare, Robert. "Psychopaths and Their Nature: Implications for the Mental Health and Criminal Justice Systems". Psychopathy: Antisocial, Criminal, and Violent Behavior. Eds. Theodore Millon, Erik Simonsen, Morten Birket-Smith, and Roger Davis. Nueva York: Guilford Press.

Ianzito, Christina. 2013. “The New 'Gone Girl'? You Decide”. AARP: Entertainment. Estados Unidos. 20 Sept. 2014. http://blog.aarp.org/2013/12/17/if-you-liked-gonegirl-check-out-these-thrillers/

Munt, Sally. 1994. Murder by the Book? Feminism and the Crime Novel. Nueva York: Routledge.

Peach, Liden. 2006. Masquerade, Crime and Fiction: Criminal Deceptions. Nueva York: Plagrave MacMillan.

SAID, Edward. 2003. Orientalism. Londres: Penguin Books.

Steinbeck, John. 1953 (2012). East of Eden. Londres: Penguin Books.

Thomas, E.M. 2012. Confessions of a Sociopath. Londres: Pan McMillan. 